\title{
Caractérisation par nanoindentation du GaN irradié par des ions uranium de grande énergie
}

\author{
Sophie Eve ${ }^{1, a}$, Florent Moisy ${ }^{2}$, Rosine Coq Germanicus ${ }^{3}$, Clara Grygiel $^{2}$, Eric Hug $^{1}$ \\ et Isabelle Monnet ${ }^{2}$
}

Reçu le 29 novembre 2016, accepté le 8 février 2017

\begin{abstract}
Résumé - Le comportement mécanique de films minces de nitrure de gallium GaN irradiés par des ions uranium accélérés a été étudié par nanoindentation. Les résultats montrent une décroissance des propriétés mécaniques du matériau par irradiation, corrélée à l'augmentation de la fluence des ions U utilisés. La perturbation croissante du réseau cristallin du GaN par irradiation conduit à une modification des mécanismes de déformation du matériau, les zones fortement désordonnées gênant le mouvement des dislocations et résultant en leur accumulation au niveau de l'interface, et à une augmentation de la dureté. Au-delà d'une fluence de $10^{13}$ ions $/ \mathrm{cm}^{2}$, le recouvrement des traces latentes créées par le passage des ions lourds conduit à une chute significative des caractéristiques mécaniques des films, et une uniformisation du comportement des matériaux irradiés aux fortes fluences.
\end{abstract}

Mots clés : GaN / irradiation / ions lourds rapides / nanoindentation / caractérisation mécanique / AFM

\begin{abstract}
The mechanical behavior of thin films of gallium nitride GaN, irradiated with swift heavy uranium ions, has been investigated by nanoindentation. Results show a decrease of the mechanical properties of the material due to irradiation, correlated to the increase of the fluency of the $U$ ions. The growing disturbance of the GaN crystalline lattice caused by irradiation results in a change in the deformation mechanisms of the material. Highly disordered zones obstruct the dislocation motion, leading to the dislocations pile-up at the film/substract interface, and a consecutive increase of the hardness. Up to a fluency of $10^{13}$ ions $/ \mathrm{cm}^{2}$, the recovering of the latent tracks created along the paths of the rapidly moving ions brings a significant drop of the mechanical properties of the GaN films, correlated with a homogenization of the behavior of the materials irradiated with elevated fluencies.
\end{abstract}

Key words: GaN / irradiation / swift heavy ions / nanoindentation / mechanical characterization / AFM

\section{Introduction}

Le nitrure de gallium (GaN) est un matériau largement utilisé pour la fabrication de composants optoélectroniques tels que des diodes électro-luminescentes (LEDs), des diodes lasers ou des détecteurs UV [1,2]. Lors d'applications en environnements radiatifs sévères, comme dans l'industrie spatiale et nucléaire, ces composants peuvent être exposés à des rayonnements ioniques de forte énergie [3,4]. Or, l'exposition de matériaux semiconducteurs aux ions de grande énergie conduit à des excitations électroniques intenses et des collisions élastiques pouvant induire des modifications des propriétés électriques, optiques et mécaniques importantes au sein du matériau [5-8]. Pour garantir leur stabilité à long terme, il est nécessaire d'identifier les défauts créés par l'irradiation de ce matériau aux ions de haute énergie, leurs mécanismes de formation et les modifications des propriétés en résultant. Pour cela nous avons étudié les caractéristiques mécaniques du GaN irradié par des ions lourds accélérés, en utilisant la nanoindentation et la microscopie à force atomique (AFM).

\footnotetext{
a Auteur de correspondance : sophie.eve@ensicaen.fr

1 ENSICAEN-CRISMAT, UMR CNRS 6508, 6 Bd Maréchal Juin, 14050 Caen, France

2 CIMAP, Normandie Université-CEA-CNRS-ENSICAEN, UMR CNRS 6252, 6 Bd Maréchal Juin, 14050 Caen, France

3 Normandie Université ENSICAEN-CRISMAT, UMR CNRS 6508, 6 Bd Maréchal Juin, 14050 Caen, France
} 


\section{2 Échantillons et moyens expérimentaux}

Les échantillons sont constitués de couches minces monocristallines de GaN épitaxiées [0001] d'une épaisseur de $3.5 \mu \mathrm{m}$, déposées sur un substrat de saphir transparent, par MOCVD (Metal Organic Chemical Vapor Deposition). Les espèces réactives sont transportées par voie gazeuse vers un substrat où elles sont simultanément pyrolisées, les températures de croissance sont dans la gamme 900-1200 ${ }^{\circ} \mathrm{C}$. Les couches de GaN sont dopées au Si (type $\mathrm{n}$ ), avec un taux de dopage de $10^{18}$ porteurs $/ \mathrm{cm}^{2}$. La densité initiale de dislocations, liée à la différence de paramètre de maille entre le substrat saphir et le GaN, est de l'ordre de $10^{9} / \mathrm{cm}^{2}$. Ces échantillons ont ensuite été irradiés au Grand Accélérateur National d'Ions Lourds (GANIL, Caen, France) sur la ligne IRRSUD. Les échantillons ont été soumis à des rayonnements d'ions uranium, d'énergie $109 \mathrm{MeV}$, avec un flux de $2 \times$ $10^{9}$ ions $/ \mathrm{cm}^{2} \mathrm{~s}$, sous incidence normale et à température ambiante. Avec ces faisceaux d'ions lourds rapides, la perte d'énergie du projectile se fait essentiellement par interaction électronique avec la cible. Le pouvoir d'arrêt des ions diminue avec la profondeur de pénétration, ainsi les ions s'implantent dans le substrat, de telle sorte qu'à forte fluence, on observe une amorphisation du saphir depuis l'interface $\mathrm{Al}_{2} \mathrm{O}_{3} / \mathrm{GaN}$. Afin d'étudier l'influence de la fluence des projectiles sur les modifications engendrées dans le matériau, et d'identifier les mécanismes d'endommagements, trois fluences ont été considérées : $5 \times 10^{12}$, $10^{13}$ et $5 \times 10^{13}$ ions $/ \mathrm{cm}^{2}$. Le GaN non irradié a été également caractérisé comme matériau de référence.

Les propriétés mécaniques du GaN vierge et irradié ont été mesurées par nanoindentation, grâce à un nanoindenteur MTS NanoXP, équipé d'une pointe Berkovich, en contrôle de déplacement, pour des profondeurs de pénétration allant de $200 \mathrm{~nm}$ à $1 \mu \mathrm{m}$ (i.e. depuis moins de $10 \%$ de l'épaisseur du film GaN, puis à travers la couche de $\mathrm{GaN}$ vers le substrat). Les essais ont été menés en mode CSM (Continuous Stiffness Measurement), neuf mesures ont été réalisées pour chaque essai. Les courbes de chargement ont été analysées et le module d'élasticité ainsi que la dureté évalués par la méthode classique d'Oliver et Pharr [9]. Finalement, les images topographiques des empreintes résiduelles ont été acquises par AFM (D3100 de Bruker) en mode tapping.

\section{Résultats et discussion}

La Figure 1a présente une courbe typique de chargedécharge obtenue par nanoindentation du GaN non irradié, à une profondeur de $200 \mathrm{~nm}$, c'est-à-dire à une valeur inférieure à $10 \%$ de l'épaisseur du film, afin de s'affranchir de l'influence éventuelle du substrat sur ces propriétés. On note un comportement élasto-plastique du GaN vierge, avec une déformation résiduelle après décharge importante, de l'ordre de $70 \%$. On observe par ailleurs la présence de discontinuités, appelées popins, sur la courbe de chargement, aux premiers temps de l'application de la charge (voir encart sur la Fig. 1a). Ces sauts soudains de déplacement sont observables à la même profondeur sur tous les essais réalisés sur les échantillons non irradiés. Les pop-ins sont caractéristiques d'une absorption ou d'une relâche d'énergie à proximité de la pointe de l'indenteur. Ils sont attribués à différents phénomènes physiques, tels que la microfissuration, des transformations de phases, une localisation de la déformation sous forme de bandes de glissement ou encore des mouvements de dislocations [10-14]. Ce phénomène est couramment observé dans les matériaux à structure hexagonale, tels que le GaN, pour lesquels il est associé au processus de déformation plastique. La caractérisation AFM de l'empreinte après indentation du film à la profondeur importante de $1 \mu \mathrm{m}$ (Fig. 1b) ne montre aucune fissure, aussi bien sous l'indenteur qu'aux sommets de l'empreinte. Ainsi le phénomène de pop-in n'est pas corrélé ici à la fissuration du GaN. De plus, le fait qu'on n'observe pas de discontinuités au cours de la décharge du matériau («pop-outs ») tendrait à exclure un changement de phases induit par indentation. Le premier pop-in apparaît ici à un déplacement et une force quasi-identiques pour chacun des essais réalisés, de l'ordre de $30-40 \mathrm{~nm}$ et $0.7 \mathrm{mN}$ respectivement, et correspondrait à un glissement des dislocations préexistantes ou nucléées lors de la pénétration de l'indeteur dans le film, lorsque la contrainte nécessaire à leur mise en mouvement est atteinte.

La Figure 2 présente l'évolution du module et de la dureté en fonction de la profondeur de pénétration. On observe que le premier pop-in s'accompagne d'une augmentation importante, suivie d'une chute soudaine de la dureté (notée par une flèche, Fig. 2b). Une chute similaire est observée sur la courbe du module, mais sa valeur reste peu affectée par ce premier pop-in. Les valeurs du module et de la dureté du GaN non irradié ont été mesurées dans le domaine stable, après le premier pop-in, et ont été évaluées à $303 \pm 6 \mathrm{GPa}$ et $20 \pm 1 \mathrm{GPa}$, respectivement. Ces valeurs sont en bon accord avec celles référencées dans la littérature pour des mesures par nanoindentation sur des films GaN épitaxiés [13,15,16]. On notera par ailleurs la présence de pop-ins de très faible amplitude se produisant lors de la poursuite de la pénétration de l'indenteur dans le GaN. Ces pop-ins conduisent à de faibles oscillations de la dureté et du module, et sont attribués à de nouvelles mises en mouvement de dislocations, mais nécessitant des niveaux d'énergie plus faibles, du fait qu'elles se produisent dans des régions déjà déformées plastiquement [11].

Après irradiation du GaN, on note une évolution des courbes du chargement (Fig. 3). Ainsi la force nécessaire pour faire pénétrer l'indenteur dans le matériau est plus faible pour les matériaux irradiés. En effet, la force à la profondeur maximum de pénétration diminue fortement par rapport au témoin lorsque le matériau a été irradié, d'autant plus que la fluence utilisée est importante. On observe par ailleurs que les matériaux irradiés aux fluences les plus fortes de $10^{13}$ et $5 \times 10^{13}$ ions $/ \mathrm{cm}^{2}$, présentent des courbes de chargement très proches. On remarque également une disparition du phénomène de 


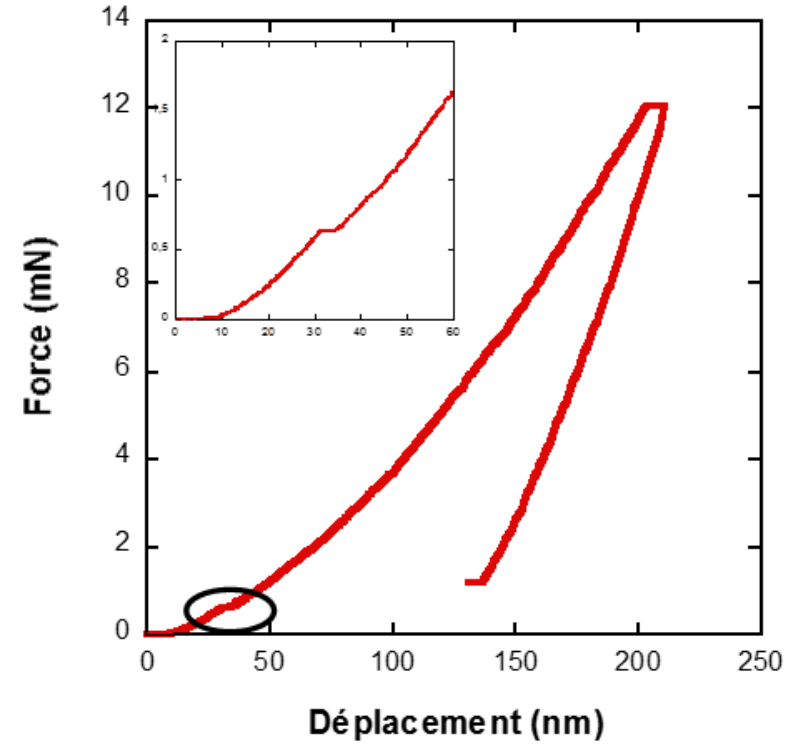

(a)

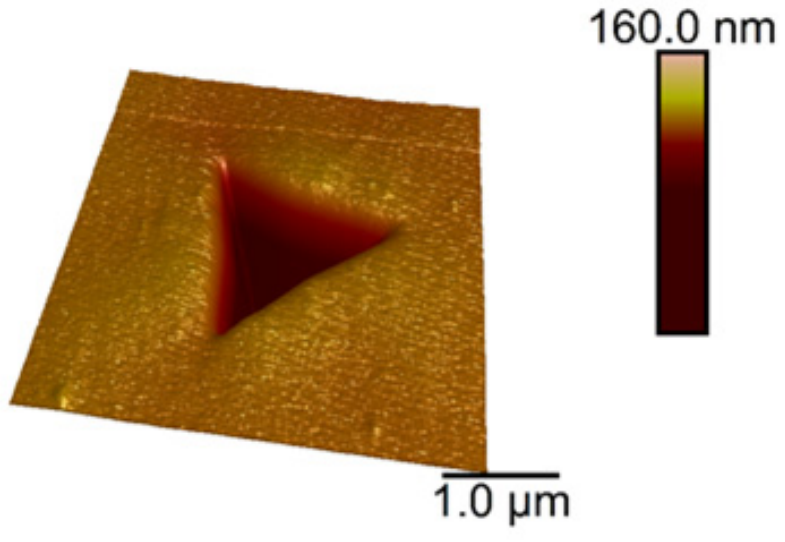

(b)

Fig. 1. (a) Courbe typique de chargement par nanoindentation du GaN non irradié pour une profondeur d'indentation de $200 \mathrm{~nm}$ (avec mise en évidence du pop-in en encart); (b) topologie 3D par AFM d'une empreinte résiduelle après décharge du GaN vierge pour une profondeur de pénétration de $1 \mu \mathrm{m}$.

Fig. 1. (a) Typical loading curve by nanoindentation of the pristine GaN with a penetration depth of $200 \mathrm{~nm}$ (with pop-in illustration in insert); (b) 3D topology by AFM of the residual print after unloading of the pristine GaN for a penetration depth of $1 \mu \mathrm{m}$.

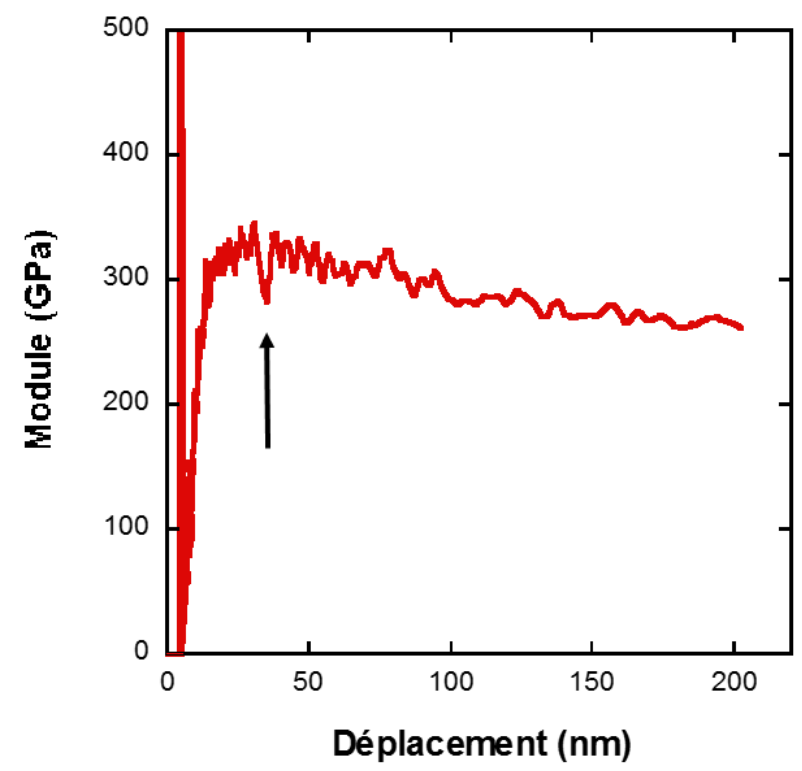

(a)

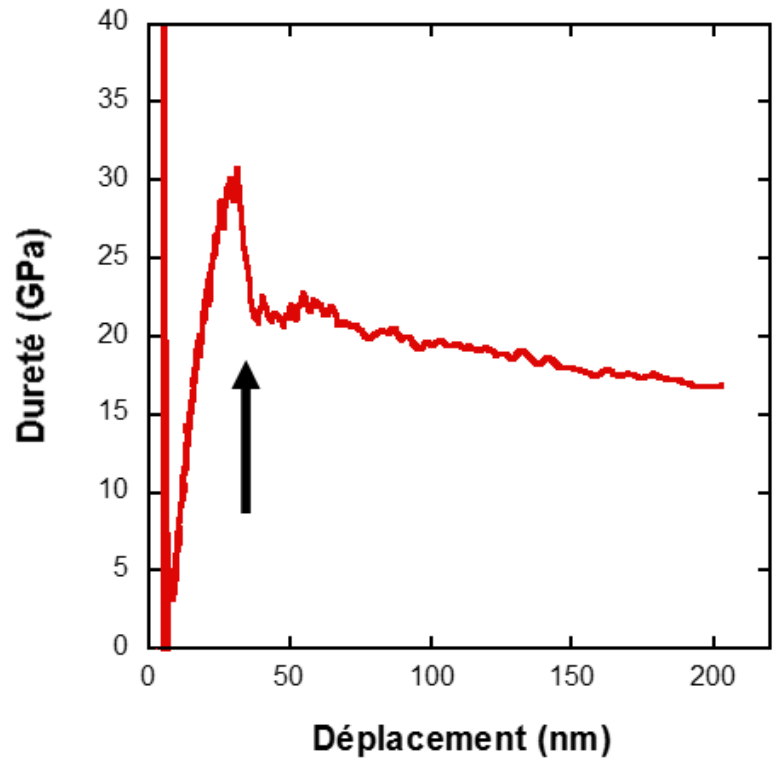

(b)

Fig. 2. Évolution (a) du module et (b) de la dureté du GaN vierge en fonction de la profondeur de pénétration.

Fig. 2. Evolution (a) of the modulus and (b) the hardness of the pristine GaN as a function of the penetration depth. 
pop-ins pour les matériaux irradiés, malgré de petits sauts de déplacement observés pour une fluence de $5 \times$ $10^{12}$ ions/cm. L'évolution des caractéristiques élastiques des matériaux irradiés suit la même tendance (Fig. 4) : on observe une diminution des valeurs du module et de la dureté des matériaux irradiés par rapport au matériau vierge.

L'observation au microscope électronique à transmission (MET) des échantillons après bombardement aux ions lourds montre l'apparition de défauts dans le film de $\mathrm{GaN}$ [17]. Il s'agit majoritairement de traces latentes discontinues, c'est-à-dire de zones endommagées, se formant le long du trajet des ions ayant pénétré rapidement la matière. Dans la littérature, on explique la formation de ces traces soit par des répulsions atomiques coulombiennes, soit par un effet thermique des ions, provoquant une fusion et un refroidissement rapide local du matériau, conduisant à son amorphisation, ou un changement de phases [18-20]. Toutefois, dans le cas du GaN, les auteurs s'accordent sur le fait que ces traces ne conduisent pas à une amorphisation totale de la trace : quelle que soit la fluence utilisée, le matériau présente ainsi un grand désordre de son réseau atomique, suite au passage des ions lourd, mais reste cristallisé $[17,21]$. L'augmentation de la fluence conduit à l'augmentation de la concentration de défauts et la croissance de la zone endommagée depuis la surface à travers l'épaisseur du film, avec une saturation à forte fluence (80\% de décanalisation observée en RBS-c [21]). En considérant un rayon de trace d'environ 2,4 nm pour les ions uranium dans le GaN [17], le recouvrement des traces latentes laissées par ces ions dans le matériau a lieu dès une fluence de $10^{13}$ ions $/ \mathrm{cm}^{2}$, et aboutit rapidement à une saturation du dommage et un désordre cristallin homogène sur une épaisseur importante de l'ordre de $2.5 \mu \mathrm{m}$ [22].

Le bombardement du GaN aux ions uranium conduit à un état cristallin clairement désordonné, qui engendre une moindre résistance mécanique. Nos résultats montrent que cette décroissance est d'autant plus importante que la fluence augmente. Au fur et à mesure que la fluence augmente, les perturbations du réseau cristallin liées au passage des ions $\mathrm{U}$ sont de plus en plus importantes, conduisant à une décroissance significative des propriétés du GaN pour les fortes fluences. Le désordre cristallin induit par l'irradiation se traduit par une augmentation des contraintes dans le matériau [8], notamment au niveau de l'interface film/substrat. La relaxation de ces contraintes par déformation plastique conduit à la nucléation et la mise en mouvement de dislocations. Or le désordre cristallin gêne fortement le déplacement des dislocations au sein du matériau, d'autant plus que la perturbation du réseau est importante. À la plus faible fluence, les mouvements des dislocations restent possibles, mais apparaissent à des profondeurs plus grandes, là où le matériau n'est pas encore totalement endommagé, tout en nécessitant une concentration de contrainte plus importante. Ceci explique la présence de faibles pop-ins sur les courbes de chargement des matériaux irradiés à faible fluence, pour des profondeurs et des forces de pénétration

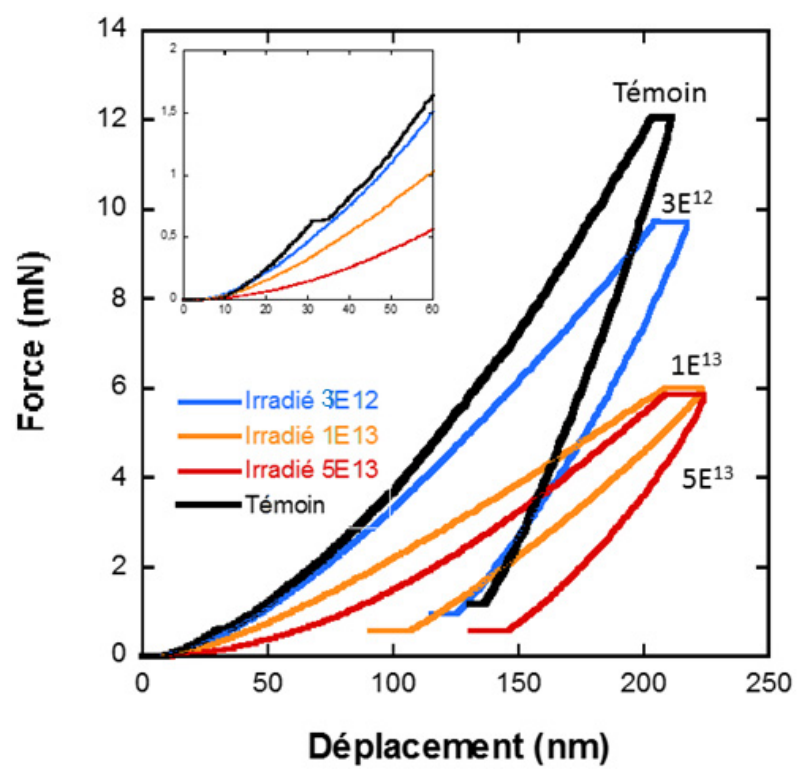

Fig. 3. Évolution (a) du module et (b) de la dureté du GaN vierge et irradié en fonction de la profondeur de pénétration, pour les différentes fluences considérées.

Fig. 3. Nanoindentation loading curves of non-irradiated and irradiated GaN using different fluencies.

plus grandes, de $40-50 \mathrm{~nm}$ et $0.8 \mathrm{mN}$ respectivement. En augmentant la fluence, les contraintes générées augmentent, résultant en une nucléation d'autant plus importante de dislocations. La croissance du désordre cristallin dans toute l'épaisseur du film conduit à l'accumulation de ces dislocations dans une zone de plus en plus petite, et donc un écrouissage significatif du GaN, ce qui pourrait expliquer le fait que la dureté de l'échantillon irradié à une fluence de $5 \times 10^{12}$ ions $/ \mathrm{cm}^{2}$ soit comparable à celle du GaN vierge [13].

Pour les fortes fluences de $10^{13}$ et $5 \times 10^{13} \mathrm{ions} / \mathrm{cm}^{2}$, le recouvrement des traces latentes résulte en une uniformisation du comportement mécanique des matériaux. Le désordre atomique devient alors très important et uniforme sur une épaisseur de $2.5 \mu \mathrm{m}$ dans le film, entrainant par ailleurs la disparition des pop-ins, et des propriétés mécaniques d'autant plus faibles. On note enfin que l'échantillon irradié avec la plus forte fluence de $5 \times 10^{13}$ ions $/ \mathrm{cm}^{2}$ présente un comportement différent des autres matériaux irradiés. Sa loi de comportement, tout comme l'évolution de son module et de sa dureté, plus stables en fonction de la profondeur de pénétration, rappelle le comportement d'un matériau amorphe. Même s'il a été montré que le GaN après bombardement ionique reste cristallin, les traces latentes créées par le passage des ions lourds peuvent comporter de petites poches de matière amorphes [21-23]. Dans un régime de recouvrement des traces, chaque nouvel ion qui traverse apporte une nouvelle partie amorphe, et augmente la fraction de matière amorphe des films, sans toutefois aboutir à la création de zones totalement amorphes [22,23] : la réponse du GaN tend alors vers celle d'un matériau amorphe. 


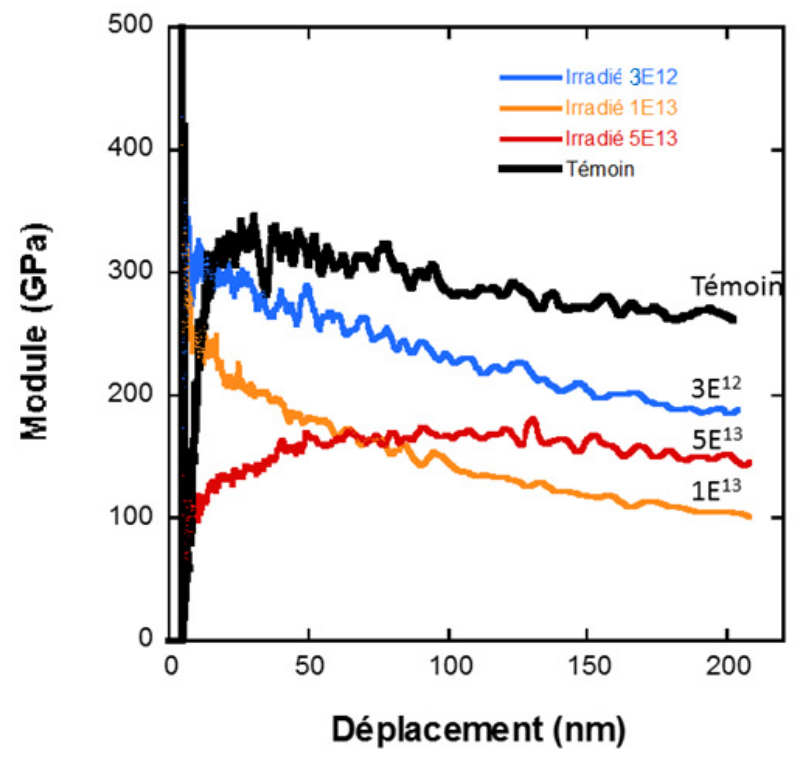

(a)

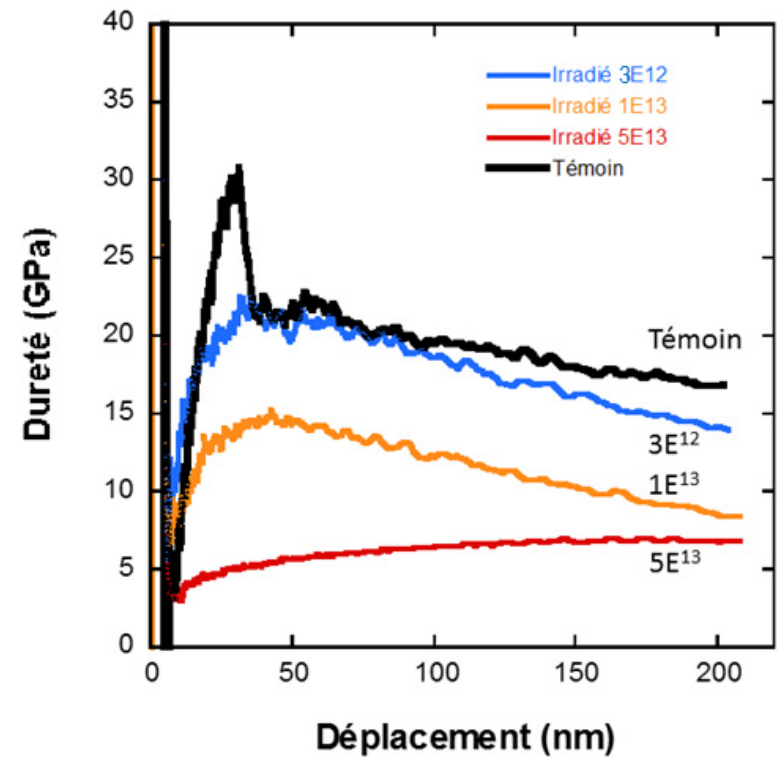

(b)

Fig. 4. Courbes de chargement par nanoindentation du GaN vierge et irradié à différentes fluences.

Fig. 4. Evolution (a) of the modulus and (b) the hardness of the non-irradiated and irradiated GaN as a function of the penetration depth, for the different investigated fluencies.
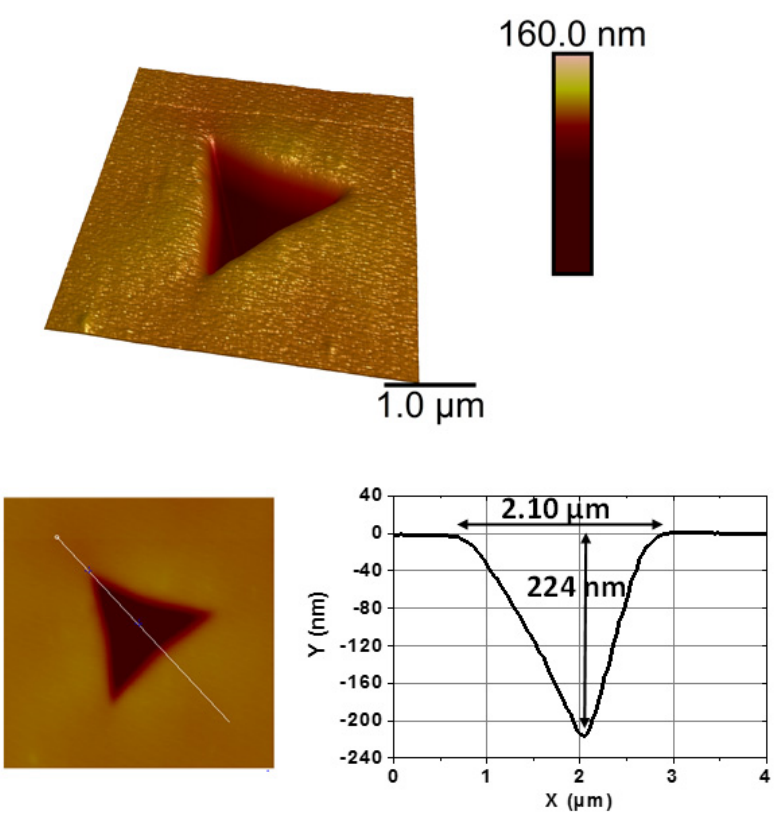

(a)
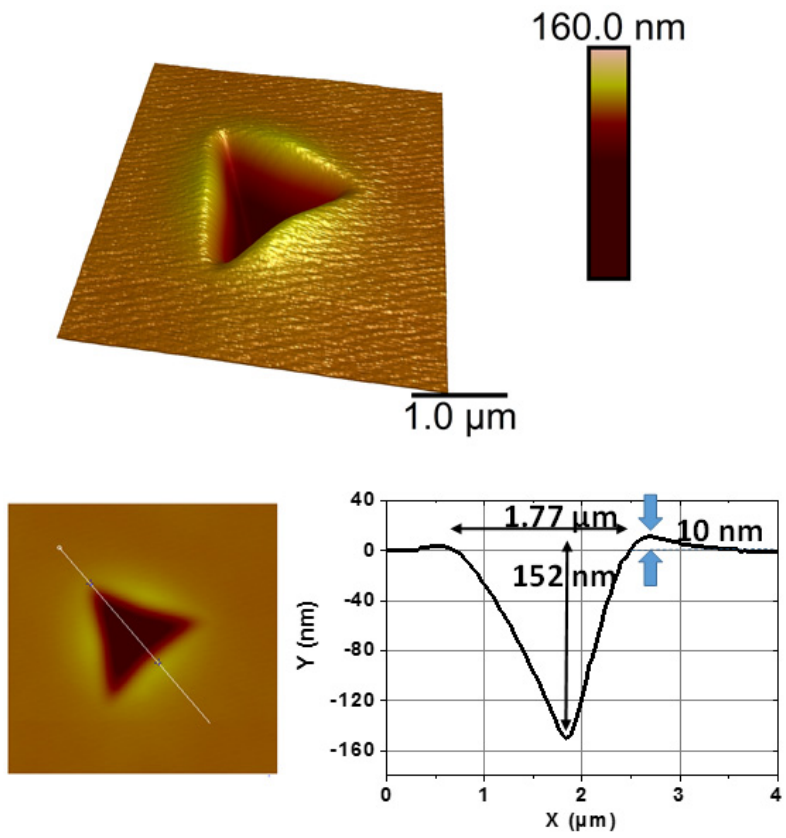

(b)

Fig. 5. Images AFM d'empreintes résiduelles après décharge du GaN (a) non-irradié et (b) irradié à une fluence de $10^{13}$ ions $/ \mathrm{cm}^{2}$ (profondeur de pénétration de $1 \mu \mathrm{m}$ ).

Fig. 5. AFM images of residual prints after unloading of the GaN (a) non-irradiated and (b) irradiated using a fluency of $10^{13}$ ions $/ \mathrm{cm}^{2}$ (penetration depth of $1 \mu \mathrm{m}$ ). 
La morphologie des indents obtenue par AFM (Fig. 5) montre un phénomène de pile-up du GaN après irradiation, avec la présence de bourrelets autour de l'empreinte résiduelle après décharge des échantillons irradiés, contrairement au GaN vierge, qui montre une forme tétragonale franche de l'empreinte Berkovich. Le pile-up est associé à un déplacement de matière par déformation plastique sous l'indenteur lors de la pénétration de la pointe, typiquement rencontré pour des systèmes « film mou sur substrat dur », des matériaux massifs présentant un fort écrouissage, ou amorphes [24]. Dans ces systèmes, le mouvement des dislocations dans le film est gêné par le substrat ou significativement freiné, cantonnant les dislocations créées lors de la pénétration de l'indenteur à proximité de la surface. Leur accumulation dans cette zone crée des contraintes résiduelles importantes, qui vont être relaxées à la décharge par mouvement de matière vers la surface. Le bombardement du GaN par des ions lourds conduit à la formation de zones présentant un fort désordre cristallin, qui interagissent avec les dislocations, en gênant significativement leur propagation vers l'intérieur du film. Les dislocations s'accumulent près de la surface, générant de fortes contraintes résiduelles dans le film de GaN, qui vont être relaxées à la décharge par la formation des bourrelets autour des indents. On note également que l'empreinte résiduelle laissée par la pointe dans le GaN non irradié a des dimensions plus importantes que le matériau irradié, ce qui traduit une déformation plastique plus importante, liée à un mouvement plus aisé des dislocations dans le matériau.

\section{Conclusion}

Les résultats de cette étude montrent qu'il est possible d'évaluer l'impact de l'irradiation par des ions lourds accélérés sur le comportement mécanique de films minces de GaN déposés sur saphir par nanoindentation. Le bombardement aux ions $\mathrm{U}$ du GaN conduit à une décroissance de ses propriétés mécaniques, liée à la production de défauts dans le matériau par le passage des ions lourds, créant des zones de grand désordre cristallin, d'autant plus étendues que la fluence utilisée augmente. Cette perturbation du réseau cristallin gêne significativement le mouvement des dislocations, diminuant la capacité du $\mathrm{GaN}$ à se déformer plastiquement. On note une diminution du module et de la dureté du GaN irradié, d'autant plus importante que la fluence augmente, que nous expliquons par un endommagement croissant du matériau, avec une uniformisation du comportement du GaN aux fortes fluences, liée au recouvrement des traces latentes pour des fluences supérieures à $10^{13}$ ions $/ \mathrm{cm}^{2}$.

\section{Références}

[1] H. Morkoc, S.N. Mohammad, Science 267 (1995) 51-55

[2] G. Fasol, Science 272 (1996) 1751-1752
[3] D.H. Lien, Y.H. Hsiao, S.G. Yang, M.L. Tsai, T.C. Wei, S.C. Lee, J.H. He, Nano Energy 11 (2015) 104-109

[4] L. Lv, J.G. Ma, Y.R. Cao, J.C. Zhang, W. Zhang, L. Li, S.R. Xu, X.H. Ma, X.T. Ren, Y. Hao, Microelectronics Reliability 51 (2011) 2168-2172

[5] V. Suresh Kumar, M. Senthil Kumar, P. Puviarasu, J. Kumar, T. Mohanty, D. Kanjilal, K. Asokan, A. Tripathi, M. Fontana, A. Camarani, Semicond. Sci. Technol. 22 (2007) 511-516

[6] K. Son, A. Liao, G. Lung, M. Gallegos, T. Hatake, R.D. Harris, L.Z. Scheick, W.D. Smythe, Nanosci. Nanotechnol. Lett. 2 (2010) 89-95

[7] J. Gou, L.Q. Zhang, C.H. Zhang, Y. Song, Y.T. Yang, J.J. Li, Y.C. Meng, H.X. Li, Nucl. Instrum. Methods Phys. Res. B 307, (2013) 89-92

[8] P.P. Hu, J. Liu, S.X. Zhang, K. Maaz, J. Zeng, H. Guo, P.F. Zhai, J.L. Duan, Y.M. Sun, M.D. Hou, Nucl. Instrum. Methods Phys. Res. B 372 (2016) 29-37

[9] W.C. Oliver, G.M. Pharr, J. Mater. Res. 7 (1992) 15641583

[10] S.J. Bull, J. Phys. D : Appl. Phys. 38 (2005) R393-R413

[11] J.E. Bradby, S.O. Kucheyev, J.S. Williams, J.W. Leu,g, M.V. Swain, P. Munroe, G. Li, M.R. Philips, Appl. Phys. Lett. 80 (2002) 383-385

[12] X.Q. Yan, X.M. Huang, S. Uda, M.W. Chen, Appl. Phys. Lett. 87 (2005) 191911

[13] S.O. Kucheyev, J.S. Williams, S.J. Pearton, Mater. Sci. Eng. 33 (2001) 51-107

[14] A.J. Haq, P.R. Munroe, M. Hoffman, P.J. Martin, A. Bendavid, Thin Solid Films 515 (2006) 1000-1004

[15] P. Kavouras, P. Komninou, T. Karakostas, Thin Solid Films 515 (2007) 3011-3018

[16] C.H. Tsai, S.R. Jian, J.Y. Juang, Appl. Surf. Sci. 254 (2008) 1997-2002

[17] F. Moisy, Étude des modifications induits dans les semiconducteurs $\mathrm{Al}_{x} \mathrm{Ga}_{1-x} \mathrm{~N}$ par irradiation aux ions lourds de haute énergie, Thèse de doctorat, Université de Caen Normandie, 2016

[18] S. Strite, H. Morkoc, J. Vac. Sci. Technol. B 10 (1992) 1237-1166

[19] S.J. Pearton, J.C. Zolper, R.J. Shul, F. Ren, J. Appl. Phys. 86 (1999) 1-78

[20] C.J. Shen, J.M. Greenberg, W.A. Schutte, E.F. van Dishoeck, Astronomy and Astrophysics 415 (2004) 203215

[21] S.O. Kucheyev, H. Timmers, J. Zou, J.S. Williams, C. Jagadish, G. Li, J. Appl. Phys. 95 (2004) 5360-5365

[22] M. Sall, I. Monnet, F. Moisy, C. Grygiel, S. JublotLeclerc, S. Della-Negra M. Toulemonde E. Balanzat, J. Mater. Sci. 50 (2015) 5214-5227

[23] F. Moisy, M. Sall, C. Grygiel, E. Balanzat, M. Boisserie, B. Lacroix, P. Simon, I. Monnet, Nucl. Instrum. Methods Phys. Res. B $\mathbf{3 8 1}$ (2016) 39-44

[24] Y.T. Cheng, C.M. Cheng, Surf. Coat. Technol. 133-134 (2000) 417-424 